\title{
An algorithm for treating asymptotically strict pseudocontractions and monotone operators
}

Mingliang Zhang*

\section{"Correspondence:}

hdzhangml@yeah.net

School of Mathematics and

Information Science, Henan

University, Kaifeng, 475000, China

\begin{abstract}
In this paper, an algorithm for treating asymptotically $\kappa$-strict pseudocontractions and monotone operators is proposed. Convergence analysis of the algorithm is investigated in the framework of Hilbert spaces.
\end{abstract}

Keywords: asymptotically $\kappa$-strict pseudocontraction; inverse-strongly monotone mapping; maximal monotone operator; fixed point

\section{Introduction-preliminaries}

In this paper, we are concerned with the problem of finding a common element in the intersection $F(T) \cap(A+B)^{-1}(0)$, where $F(T)$ denotes the fixed point set of the mapping $T$ and $(A+B)^{-1}(0)$ denotes the zero point set of the sum of the operator $A$ and the operator $B$.

The motivation for the common element problem is mainly due to its possible applications to mathematical modeling of concrete complex problems. The common element problems include mini-max problems, complementarity problems, equilibrium problems, common fixed point problems and variational inequalities as special cases; see, for example, [1-35] and the references therein.

Throughout the article, we always assume that $H$ is a real Hilbert space with the inner product $\langle\cdot, \cdot\rangle$ and the norm $\|\cdot\|$, respectively. Let $C$ be a nonempty closed convex subset of $H$, and let $\operatorname{Proj}_{C}$ be the metric projection from $H$ onto $C$.

Let $A: C \rightarrow H$ be a mapping. $A^{-1}(0)$ stands for the zero point set of $A$; that is, $A^{-1}(0):=$ $\{x \in C: A x=0\}$. Recall that $A$ is said to be monotone iff

$$
\langle A x-A y, x-y\rangle \geq 0, \quad \forall x, y \in C .
$$

$A$ is said to be $\alpha$-strongly monotone iff there exists a constant $\alpha>0$ such that

$$
\langle A x-A y, x-y\rangle \geq \alpha\|x-y\|^{2}, \quad \forall x, y \in C .
$$

$A$ is said to be $\alpha$-inverse-strongly monotone iff there exists a constant $\alpha>0$ such that

$$
\langle A x-A y, x-y\rangle \geq \alpha\|A x-A y\|^{2}, \quad \forall x, y \in C .
$$

O2014 Zhang; licensee Springer. This is an Open Access article distributed under the terms of the Creative Commons Attribution License (http://creativecommons.org/licenses/by/2.0), which permits unrestricted use, distribution, and reproduction in any medium, provided the original work is properly cited. 
It is not hard to see that $\alpha$-inverse-strongly monotone mappings are Lipschitz continuous. Indeed, we have

$$
\alpha\|A x-A y\|^{2} \leq\langle A x-A y, x-y\rangle \leq\|A x-A y\|\|x-y\| .
$$

This shows that $\|A x-A y\| \leq \frac{1}{\alpha}\|x-y\|$.

Recall that the classical variational inequality, denoted by $V I(C, A)$, is to find $u \in C$ such that

$$
\langle A u, v-u\rangle \geq 0, \quad \forall v \in C
$$

One can see that the variational inequality (1.1) is equivalent to a fixed point problem of the mapping $\operatorname{Proj}_{C}(I-r A)$, where $I$ is the identity and $r$ is some positive real number. The element $u \in C$ is a solution of the variational inequality (1.1) iff $u \in C$ satisfies the equation $u=P_{C}(u-r A u)$. This alternative equivalent formulation has played a significant role in the studies of variational inequalities and related optimization problems.

A multivalued operator $B: H \rightarrow 2^{H}$ with the domain $D(B)=\{x \in H: B x \neq \emptyset\}$ and the range $R(B)=\{B x: x \in D(B)\}$ is said to be monotone if for $x_{1} \in D(B), x_{2} \in D(B), y_{1} \in B x_{1}$ and $y_{2} \in B x_{2}$, we have $\left\langle x_{1}-x_{2}, y_{1}-y_{2}\right\rangle \geq 0$. A monotone operator $B$ is said to be maximal if its graph $G(B)=\{(x, y): y \in B x\}$ is not properly contained in the graph of any other monotone operator. Let $I$ denote the identity operator on $H$ and $B: H \rightarrow 2^{H}$ be a maximal monotone operator. Then we can define, for each $r>0$, a nonexpansive single-valued mapping $J_{r}$ : $H \rightarrow H$ by $J_{r}=(I+r B)^{-1}$. It is called the resolvent of $B$. We know that $B^{-1} 0=F\left(J_{r}\right)$ for all $r>0$ and $J_{r}$ is firmly nonexpansive.

Let $T: C \rightarrow C$ be a mapping. In this paper, we use $F(T)$ to denote the fixed point set of $T$; that is, $F(T):=\{x \in C: x=T x\}$. Recall that $T$ is said to be nonexpansive iff

$$
\|T x-T y\| \leq\|x-y\|, \quad \forall x, y \in C \text {. }
$$

$T$ is said to be asymptotically nonexpansive iff there exists a sequence $\left\{k_{n}\right\} \subset[1, \infty)$ such that

$$
\left\|T^{n} x-T^{n} y\right\| \leq k_{n}\|x-y\|, \quad \forall x, y \in C, n \geq 1
$$

$T$ is said to be a $\kappa$-strict pseudocontraction iff there exists a constant $\kappa \in[0,1)$ such that

$$
\|T x-T y\|^{2} \leq\|x-y\|^{2}+\kappa\|(x-T x)-(y-T y)\|^{2}, \quad \forall x, y \in C .
$$

Note that the class of $\kappa$-strict pseudocontractions strictly includes the class of nonexpansive mappings as a special case. That is, $T$ is nonexpansive iff the coefficient $\kappa=0 . T$ is said to be an asymptotically $\kappa$-strict pseudocontraction iff there exist a constant $\kappa \in[0,1)$ and a sequence $\left\{k_{n}\right\}$ in $[1, \infty)$ such that

$$
\left\|T^{n} x-T^{n} y\right\|^{2} \leq k_{n}\|x-y\|^{2}+\kappa\left\|\left(x-T^{n} x\right)-\left(y-T^{n} y\right)\right\|^{2}, \quad \forall x, y \in C, n \geq 1 .
$$

Note that the class of asymptotically $\kappa$-strict pseudocontractions strictly includes the class of asymptotically nonexpansive mappings as a special case. That is, $T$ is asymptotically nonexpansive iff the coefficient $\kappa=0$. 
In [24], Kamimura and Takahashi investigated the problem of finding zero points of a maximal monotone operator based on the following iterative algorithm:

$$
x_{1} \in H, \quad x_{n+1}=\alpha_{n} x+\left(1-\alpha_{n}\right) J_{r_{n}} x_{n}, \quad \forall n \geq 1,
$$

where $\left\{\alpha_{n}\right\}$ is a sequence in $(0,1),\left\{r_{n}\right\}$ is a positive real number sequence, $B: H \rightarrow 2^{H}$ is maximal monotone and $J_{r_{n}}=\left(I+r_{n} B\right)^{-1}$. It is proved that the sequence $\left\{x_{n}\right\}$ generated in (1.2) converges strongly to some $z \in B^{-1}(0)$ provided that the control sequence satisfies some restrictions. Further, using this result, they also investigated the case that $B=\partial f$, where $f: H \rightarrow(-\infty, \infty]$ is a proper lower semicontinuous convex function. Convergence theorems are established in the framework of real Hilbert spaces; for more details, see [24].

Recently, Takahashi et al. studied zero point problems of the sum of two monotone mappings and fixed point problems of a nonexpansive mapping based on the following iterative algorithm:

$$
\left\{\begin{array}{l}
x_{1} \in C, \\
y_{n}=\alpha_{n} x+\left(1-\alpha_{n}\right) J_{r_{n}}\left(x_{n}-r_{n} A x_{n}\right), \\
x_{n+1}=\beta_{n} x_{n}+\left(1-\beta_{n}\right) T y_{n}, \quad \forall n \geq 1,
\end{array}\right.
$$

where $\left\{\alpha_{n}\right\}$ and $\left\{\beta_{n}\right\}$ are real number sequences in $(0,1),\left\{r_{n}\right\}$ is a positive sequence, $T: C \rightarrow C$ is a nonexpansive mapping and $A: C \rightarrow H$ is an inverse-strongly monotone mapping. It is proved that the sequence $\left\{x_{n}\right\}$ generated in (1.3) converges strongly to some $z \in(A+B)^{-1}(0) \cap F(S)$ provided that the control sequence satisfies some restrictions; for more details, see [2].

Motivated by the above results, we investigate fixed point problems of asymptotically strict pseudocontractions and zero point problems of the sum of two monotone mappings. In order to state our main results, we need the following tools.

Recall that a space is said to satisfy Opial's condition [36] if, for any sequence $\left\{x_{n}\right\} \subset H$ with $x_{n} \rightarrow x$, where $\rightarrow$ denotes the weak convergence, the inequality

$$
\liminf _{n \rightarrow \infty}\left\|x_{n}-x\right\|<\liminf _{n \rightarrow \infty}\left\|x_{n}-y\right\|
$$

holds for every $y \in H$ with $y \neq x$. Indeed, the above inequality is equivalent to the following:

$$
\limsup _{n \rightarrow \infty}\left\|x_{n}-x\right\|<\limsup _{n \rightarrow \infty}\left\|x_{n}-y\right\| .
$$

Lemma 1.1 [2] Let $\left\{x_{n}\right\}$ and $\left\{y_{n}\right\}$ be bounded sequences in a Banach space $X$, and let $\beta_{n}$ be a sequence in $[0,1]$ with $0<\liminf _{n \rightarrow \infty} \beta_{n} \leq \lim \sup _{n \rightarrow \infty} \beta_{n}<1$. Suppose that $x_{n+1}=$ $\left(1-\beta_{n}\right) y_{n}+\beta_{n} x_{n}$ for all integers $n \geq 0$ and

$$
\limsup _{n \rightarrow \infty}\left(\left\|y_{n+1}-y_{n}\right\|-\left\|x_{n+1}-x_{n}\right\|\right) \leq 0 .
$$

Then $\lim _{n \rightarrow \infty}\left\|y_{n}-x_{n}\right\|=0$.

Lemma 1.2 [37] Let $C$ be a nonempty, closed and convex subset of $H$, let $A: C \rightarrow H$ be a mapping, and let $B: H \rightrightarrows H$ be a maximal monotone operator. Then $F\left(J_{r}(I-r A)\right)=(A+$ $B)^{-1}(0)$. 
Lemma 1.3 [38] Assume that $\left\{\alpha_{n}\right\}$ is a sequence of nonnegative real numbers such that

$$
\alpha_{n+1} \leq\left(1-\gamma_{n}\right) \alpha_{n}+\delta_{n}
$$

where $\left\{\gamma_{n}\right\}$ is a sequence in $(0,1)$ and $\left\{\delta_{n}\right\}$ is a sequence such that

(i) $\sum_{n=1}^{\infty} \gamma_{n}=\infty$;

(ii) $\lim \sup _{n \rightarrow \infty} \delta_{n} / \gamma_{n} \leq 0$ or $\sum_{n=1}^{\infty}\left|\delta_{n}\right|<\infty$.

Then $\lim _{n \rightarrow \infty} \alpha_{n}=0$.

Lemma 1.4 [39] Let $C$ be a nonempty, closed and convex subset of $H$. Let $T: C \rightarrow C$ be an asymptotically strict pseudocontraction. Then $T$ is Lipschitz continuous and $I-T$ is demiclosed at zero.

\section{Main results}

Theorem 2.1 Let $C$ be a nonempty closed convex subset of H. Let $T: C \rightarrow C$ be an asymptotically $\kappa$-strict pseudocontraction. Let $A: C \rightarrow H$ be an $\alpha$-inverse-strongly monotone mapping, and let $B$ be a maximal monotone operator on $H$. Assume that $F(T) \cap(A+$ $B)^{-1}(0) \neq \emptyset$. Let $\left\{\alpha_{n}\right\},\left\{\beta_{n}\right\}$ and $\left\{\gamma_{n}\right\}$ be real number sequences in $(0,1)$. Let $J_{r_{n}}=\left(I+r_{n} B\right)^{-1}$, where $\left\{r_{n}\right\}$ is a positive real number sequence. Let $\left\{x_{n}\right\}$ be a sequence in $C$ generated by: $x_{1} \in C$ is chosen arbitrarily and

$$
\left\{\begin{array}{l}
z_{n}=\operatorname{Proj}_{C}\left(\alpha_{n} u+\left(1-\alpha_{n}\right) J_{r_{n}}\left(x_{n}-r_{n} A x_{n}\right)\right), \\
x_{n+1}=\beta_{n} x_{n}+\left(1-\beta_{n}\right)\left(\gamma_{n} z_{n}+\left(1-\gamma_{n}\right) T^{n} z_{n}\right), \quad \forall n \geq 1 .
\end{array}\right.
$$

Assume that the sequences $\left\{\alpha_{n}\right\},\left\{\beta_{n}\right\},\left\{\gamma_{n}\right\}$ and $\left\{r_{n}\right\}$ satisfy the following restrictions:

(a) $0<a \leq r_{n} \leq b<2 \alpha, \lim _{n \rightarrow \infty}\left|r_{n+1}-r_{n}\right|=0$;

(b) $\lim _{n \rightarrow \infty} \alpha_{n}=0, \sum_{n=1}^{\infty} \alpha_{n}=\infty$;

(c) $0<c \leq \beta_{n} \leq d<1$;

(d) $\kappa \leq \gamma_{n} \leq e<1, \lim _{n \rightarrow \infty}\left|\gamma_{n+1}-\gamma_{n}\right|=0$,

where $a, b, c, d$ and e are some real numbers. If $T$ is asymptotically regular, then the sequence $\left\{x_{n}\right\}$ converges strongly to some point $\bar{x}$, where $\bar{x}=P_{F(T) \cap(A+B)^{-1}(0)} u$.

Proof First, we show that the mapping $I-r_{n} A$ is nonexpansive. Indeed, we have

$$
\begin{aligned}
\|( & \left(I-r_{n} A\right) x-\left(I-r_{n} A\right) y \|^{2} \\
& =\|x-y\|^{2}-2 r_{n}\langle x-y, A x-A y\rangle+r_{n}{ }^{2}\|A x-A y\|^{2} \\
& \leq\|x-y\|^{2}-r_{n}\left(2 \alpha-r_{n}\right)\|A x-A y\|^{2} .
\end{aligned}
$$

It follows from Restriction (a) that $I-r_{n} A$ is nonexpansive. Put $y_{n}=\gamma_{n} z_{n}+\left(1-\gamma_{n}\right) T^{n} z_{n}$ and fix $p \in F(T) \cap(A+B)^{-1}(0)$. It follows from Lemma 1.2 that

$$
\begin{aligned}
\left\|z_{n}-p\right\| & =\left\|\operatorname{Proj}_{C}\left(\alpha_{n} u+\left(1-\alpha_{n}\right) J_{r_{n}}\left(x_{n}-r_{n} A x_{n}\right)\right)-p\right\| \\
& \leq \alpha_{n}\|u-p\|+\left(1-\alpha_{n}\right)\left\|J_{r_{n}}\left(x_{n}-r_{n} A x_{n}\right)-p\right\| \\
& \leq \alpha_{n}\|u-p\|+\left(1-\alpha_{n}\right)\left\|x_{n}-p\right\| .
\end{aligned}
$$


In view of Restriction $(\mathrm{d})$, we find that

$$
\begin{aligned}
\left\|y_{n}-p\right\|^{2} \leq & \left\|\gamma_{n}\left(z_{n}-p\right)+\left(1-\gamma_{n}\right)\left(T^{n} z_{n}-T^{n} p\right)\right\|^{2} \\
= & \gamma_{n}\left\|z_{n}-p\right\|^{2}+\left(1-\gamma_{n}\right)\left\|T^{n} z_{n}-T^{n} p\right\|^{2} \\
& \quad-\gamma_{n}\left(1-\gamma_{n}\right)\left\|\left(z_{n}-p\right)-\left(T^{n} z_{n}-T^{n} p\right)\right\|^{2} \\
\leq & \gamma_{n}\left\|z_{n}-p\right\|^{2}+\left(1-\gamma_{n}\right)\left(\left\|z_{n}-p\right\|^{2}+\kappa\left\|\left(z_{n}-p\right)-\left(T^{n} z_{n}-T^{n} p\right)\right\|^{2}\right) \\
& \quad-\gamma_{n}\left(1-\gamma_{n}\right)\left\|\left(z_{n}-p\right)-\left(T^{n} z_{n}-T^{n} p\right)\right\|^{2} \\
= & \left\|z_{n}-p\right\|^{2}-\left(1-\gamma_{n}\right)\left(\gamma_{n}-\kappa\right)\left\|\left(z_{n}-p\right)-\left(T^{n} z_{n}-T^{n} p\right)\right\|^{2} \\
\leq & \left\|z_{n}-p\right\|^{2} .
\end{aligned}
$$

Substituting (2.1) into (2.2), we obtain that

$$
\begin{aligned}
\left\|x_{n+1}-p\right\| & \leq \beta_{n}\left\|x_{n}-p\right\|+\left(1-\beta_{n}\right)\left\|y_{n}-p\right\| \\
& \leq \beta_{n}\left\|x_{n}-p\right\|+\left(1-\beta_{n}\right)\left(\alpha_{n}\|u-p\|+\left(1-\alpha_{n}\right)\left\|x_{n}-p\right\|\right) \\
& \leq\left(1-\alpha_{n}\left(1-\beta_{n}\right)\right)\left\|x_{n}-p\right\|+\alpha_{n}\left(1-\beta_{n}\right)\|u-p\| .
\end{aligned}
$$

Putting $M=\max \left\{\left\|x_{1}-p\right\|,\|u-p\|\right\}$, we find that $\left\|x_{n}-p\right\| \leq M$ for all $n \geq 1$. Indeed, it is clear that $\left\|x_{1}-p\right\| \leq M$. Suppose that $\left\|x_{m}-p\right\| \leq M$ for some positive integer $m$. It follows that

$$
\begin{aligned}
\left\|x_{m+1}-p\right\| & \leq\left(1-\alpha_{m}\left(1-\beta_{m}\right)\right)\left\|x_{m}-p\right\|+\alpha_{m}\left(1-\beta_{m}\right)\|u-p\| \\
& \leq\left(1-\alpha_{m}\left(1-\beta_{m}\right)\right) M+\alpha_{m}\left(1-\beta_{n}\right) M \\
& =M
\end{aligned}
$$

This finds that $\left\{x_{n}\right\}$ is bounded. Putting $\rho_{n}=J_{r_{n}}\left(x_{n}-r_{n} A x_{n}\right)$, we find that

$$
\begin{aligned}
\left\|\rho_{n+1}-\rho_{n}\right\| \leq & \left\|J_{r_{n+1}}\left(x_{n+1}-r_{n+1} A x_{n+1}\right)-J_{r_{n+1}}\left(x_{n}-r_{n} A x_{n}\right)\right\| \\
& +\left\|J_{r_{n+1}}\left(x_{n}-r_{n} A x_{n}\right)-J_{r_{n}}\left(x_{n}-r_{n} A x_{n}\right)\right\| \\
\leq & \left\|\left(x_{n+1}-r_{n+1} A x_{n+1}\right)-\left(x_{n}-r_{n} A x_{n}\right)\right\| \\
& +\left\|J_{r_{n+1}}\left(x_{n}-r_{n} A x_{n}\right)-J_{r_{n}}\left(x_{n}-r_{n} A x_{n}\right)\right\| \\
\leq & \left\|x_{n+1}-x_{n}\right\|+\left|r_{n+1}-r_{n}\right|\left\|A x_{n}\right\| \\
& +\left\|J_{r_{n+1}}\left(x_{n}-r_{n} A x_{n}\right)-J_{r_{n}}\left(x_{n}-r_{n} A x_{n}\right)\right\| .
\end{aligned}
$$

On the other hand, we have

$$
\begin{aligned}
\left\|z_{n+1}-z_{n}\right\| & \leq\left\|\left(\alpha_{n+1} u+\left(1-\alpha_{n+1}\right) \rho_{n+1}\right)-\left(\alpha_{n} u+\left(1-\alpha_{n}\right) \rho_{n}\right)\right\| \\
& \leq\left(1-\alpha_{n+1}\right)\left\|\rho_{n+1}-\rho_{n}\right\|+\left|\alpha_{n+1}-\alpha_{n}\right|\left\|\rho_{n}-u\right\| .
\end{aligned}
$$


Substituting (2.3) into (2.4), we find that

$$
\begin{aligned}
\left\|z_{n+1}-z_{n}\right\| \leq & \left\|\left(\alpha_{n+1} u+\left(1-\alpha_{n+1}\right) \rho_{n+1}\right)-\left(\alpha_{n} u+\left(1-\alpha_{n}\right) \rho_{n}\right)\right\| \\
\leq & \left(1-\alpha_{n+1}\right)\left\|x_{n+1}-x_{n}\right\|+\left|r_{n+1}-r_{n}\right|\left\|A x_{n}\right\|+\left|\alpha_{n+1}-\alpha_{n}\right|\left\|\rho_{n}-u\right\| \\
& +\left(1-\alpha_{n+1}\right)\left\|J_{r_{n+1}}\left(x_{n}-r_{n} A x_{n}\right)-J_{r_{n}}\left(x_{n}-r_{n} A x_{n}\right)\right\| .
\end{aligned}
$$

Put $\xi_{n}=x_{n}-r_{n} A x_{n}$. Since $B$ is monotone, we find that

$$
\left\langle J_{r_{n+1}} \xi_{n}-J_{r_{n}} \xi_{n}, \frac{\xi_{n}-J_{r_{n+1}} \xi_{n}}{r_{n+1}}-\frac{\xi_{n}-J_{r_{n}} \xi_{n}}{r_{n}}\right\rangle \geq 0
$$

It follows that $\left\langle J_{r_{n+1}} \xi_{n}-J_{r_{n}} \xi_{n},\left(1-\frac{r_{n+1}}{r_{n}}\right)\left(\xi_{n}-J_{r_{n}} \xi_{n}\right)\right\rangle \geq\left\|J_{r_{n+1}} \xi_{n}-J_{r_{n}} \xi_{n}\right\|^{2}$. This yields that $\mid r_{n+1}-$ $r_{n} \mid\left\|\xi_{n}-J_{r_{n}} \xi_{n}\right\| \geq r_{n}\left\|J_{r_{n+1}} \xi_{n}-J_{r_{n}} \xi_{n}\right\|$. This combines with (2.5) to yield that

$$
\begin{aligned}
\left\|z_{n+1}-z_{n}\right\| \leq & \left(1-\alpha_{n+1}\right)\left\|x_{n+1}-x_{n}\right\|+\left|r_{n+1}-r_{n}\right|\left\|A x_{n}\right\| \\
& +\left|\alpha_{n+1}-\alpha_{n}\right|\left\|\rho_{n}-u\right\|+\frac{\left|r_{n+1}-r_{n}\right|}{r_{n}}\left\|\xi_{n}-J_{r_{n}} \xi_{n}\right\| .
\end{aligned}
$$

On the other hand, we have

$$
\begin{gathered}
\left\|y_{n+1}-y_{n}\right\| \leq \gamma_{n+1}\left\|z_{n+1}-z_{n}\right\|+\left|\gamma_{n+1}-\gamma_{n}\right|\left\|z_{n}-T^{n} z_{n}\right\| \\
+\left(1-\gamma_{n+1}\right)\left\|T^{n+1} z_{n+1}-T^{n} z_{n}\right\| .
\end{gathered}
$$

Substituting (2.6) into (2.7), we find that

$$
\begin{aligned}
& \left\|y_{n+1}-y_{n}\right\|-\left\|x_{n+1}-x_{n}\right\| \\
& \quad \leq\left|r_{n+1}-r_{n}\right|\left\|A x_{n}\right\|+\left|\alpha_{n+1}-\alpha_{n}\right|\left\|\rho_{n}-u\right\| \\
& \quad+\frac{\left|r_{n+1}-r_{n}\right|}{r_{n}}\left\|\xi_{n}-J_{r_{n}} \xi_{n}\right\|+\left|\gamma_{n+1}-\gamma_{n}\right|\left\|z_{n}-T^{n} z_{n}\right\| \\
& \quad+\left(1-\gamma_{n+1}\right)\left\|T^{n+1} z_{n+1}-T^{n} z_{n}\right\| .
\end{aligned}
$$

It follows from Restrictions (a), (c) and (d) that

$$
\limsup _{n \rightarrow \infty}\left(\left\|y_{n+1}-y_{n}\right\|-\left\|x_{n+1}-x_{n}\right\|\right) \leq 0
$$

It follows from Lemma 1.1 that $\lim _{n \rightarrow \infty}\left\|y_{n}-x_{n}\right\|=0$. Since $x_{n+1}-x_{n}=\left(1-\beta_{n}\right)\left(y_{n}-x_{n}\right)$, we find that $\lim _{n \rightarrow \infty}\left\|x_{n+1}-x_{n}\right\|=0$. Notice that

$$
\begin{aligned}
& \left\|J_{r_{n}}\left(I-r_{n} A\right) x_{n}-J_{r_{n}}\left(I-r_{n} A\right) p\right\|^{2} \\
& \quad=\left\|\left(x_{n}-p\right)-r_{n}\left(A x_{n}-A p\right)\right\|^{2} \\
& \quad=\left\|x_{n}-p\right\|^{2}-2 r_{n}\left\langle x_{n}-p, A x_{n}-A p\right\rangle+r_{n}{ }^{2}\left\|A x_{n}-A p\right\|^{2} \\
& \quad \leq\left\|x_{n}-p\right\|^{2}-r_{n}\left(2 \alpha-r_{n}\right)\left\|A x_{n}-A p\right\|^{2} .
\end{aligned}
$$


Since the norm is convex, we see from (2.2) and (2.8) that

$$
\begin{aligned}
\left\|x_{n+1}-p\right\|^{2} \leq & \beta_{n}\left\|x_{n}-p\right\|^{2}+\left(1-\beta_{n}\right)\left\|y_{n}-p\right\|^{2} \\
\leq & \beta_{n}\left\|x_{n}-p\right\|^{2}+\left(1-\beta_{n}\right)\left\|z_{n}-p\right\|^{2} \\
\leq & \beta_{n}\left\|x_{n}-p\right\|^{2}+\left(1-\beta_{n}\right)\left\|\alpha_{n}(u-p)+\left(1-\alpha_{n}\right)\left(J_{r_{n}}\left(x_{n}-r_{n} A x_{n}\right)-p\right)\right\|^{2} \\
\leq & \beta_{n}\left\|x_{n}-p\right\|^{2}+\alpha_{n}\left(1-\beta_{n}\right)\|u-p\|^{2} \\
& \quad+\left(1-\alpha_{n}\right)\left(1-\beta_{n}\right)\left\|J_{r_{n}}\left(x_{n}-r_{n} A x_{n}\right)-p\right\|^{2} \\
\leq & \left\|x_{n}-p\right\|^{2}+\alpha_{n}\|u-p\|^{2}-r_{n}\left(2 \alpha-r_{n}\right)\left(1-\alpha_{n}\right)\left(1-\beta_{n}\right)\left\|A x_{n}-A p\right\|^{2} .
\end{aligned}
$$

This yields that

$$
\begin{aligned}
& r_{n}\left(2 \alpha-r_{n}\right)\left(1-\alpha_{n}\right)\left(1-\beta_{n}\right)\left\|A x_{n}-A p\right\|^{2} \\
& \quad \leq\left\|x_{n}-p\right\|^{2}+\alpha_{n}\|u-p\|^{2}-\left\|x_{n+1}-p\right\|^{2} \\
& \quad \leq\left(\left\|x_{n}-p\right\|+\left\|x_{n+1}-p\right\|\right)\left\|x_{n+1}-x_{n}\right\|+\alpha_{n}\|u-p\|^{2} .
\end{aligned}
$$

In view of Restrictions (a), (b) and (c), we obtain that

$$
\lim _{n \rightarrow \infty}\left\|A x_{n}-A p\right\|=0
$$

Notice that

$$
\begin{aligned}
\left\|\rho_{n}-p\right\|^{2}= & \left\|J_{r_{n}}\left(x_{n}-r_{n} A x_{n}\right)-J_{r_{n}}\left(p-r_{n} A p\right)\right\|^{2} \\
\leq & \left\langle\left(x_{n}-r_{n} A x_{n}\right)-\left(p-r_{n} A p\right), \rho_{n}-p\right\rangle \\
= & \frac{1}{2}\left(\left\|\left(x_{n}-r_{n} A x_{n}\right)-\left(p-r_{n} A p\right)\right\|^{2}+\left\|\rho_{n}-p\right\|^{2}\right. \\
& \left.-\left\|\left(x_{n}-r_{n} A x_{n}\right)-\left(p-r_{n} A p\right)-\left(\rho_{n}-p\right)\right\|^{2}\right) \\
\leq & \frac{1}{2}\left(\left\|x_{n}-p\right\|^{2}+\left\|\rho_{n}-p\right\|^{2}-\left\|x_{n}-\rho_{n}-r_{n}\left(A x_{n}-A p\right)\right\|^{2}\right) \\
\leq & \frac{1}{2}\left(\left\|x_{n}-p\right\|^{2}+\left\|\rho_{n}-p\right\|^{2}-\left\|x_{n}-\rho_{n}\right\|^{2}-r_{n}^{2}\left\|A x_{n}-A p\right\|^{2}\right. \\
& \left.+2 r_{n}\left\|x_{n}-\rho_{n}\right\|\left\|A x_{n}-A p\right\|\right) \\
\leq & \frac{1}{2}\left(\left\|x_{n}-p\right\|^{2}+\left\|\rho_{n}-p\right\|^{2}-\left\|x_{n}-\rho_{n}\right\|^{2}+2 r_{n}\left\|x_{n}-\rho_{n}\right\|\left\|A x_{n}-A p\right\|\right) .
\end{aligned}
$$

It follows that

$$
\left\|\rho_{n}-p\right\|^{2} \leq\left\|x_{n}-p\right\|^{2}-\left\|x_{n}-\rho_{n}\right\|^{2}+2 r_{n}\left\|x_{n}-\rho_{n}\right\|\left\|A x_{n}-A p\right\| .
$$

This yields that

$$
\begin{aligned}
\left\|z_{n}-p\right\|^{2} & \leq \alpha_{n}\|u-p\|^{2}+\left(1-\alpha_{n}\right)\left\|\rho_{n}-p\right\|^{2} \\
& \leq \alpha_{n}\|u-p\|^{2}+\left\|x_{n}-p\right\|^{2}-\left(1-\alpha_{n}\right)\left\|x_{n}-\rho_{n}\right\|^{2}+2 r_{n}\left\|x_{n}-\rho_{n}\right\|\left\|A x_{n}-A p\right\| .
\end{aligned}
$$


It follows from (2.2) that

$$
\begin{aligned}
\left\|x_{n+1}-p\right\|^{2} \leq & \beta_{n}\left\|x_{n}-p\right\|^{2}+\left(1-\beta_{n}\right)\left\|y_{n}-p\right\|^{2} \\
\leq & \beta_{n}\left\|x_{n}-p\right\|^{2}+\left(1-\beta_{n}\right)\left\|z_{n}-p\right\|^{2} \\
\leq & \left\|x_{n}-p\right\|^{2}+\alpha_{n}\|u-p\|^{2}-\left(1-\alpha_{n}\right)\left(1-\beta_{n}\right)\left\|x_{n}-\rho_{n}\right\|^{2} \\
& +2 r_{n}\left(1-\beta_{n}\right)\left\|x_{n}-\rho_{n}\right\|\left\|A x_{n}-A p\right\| .
\end{aligned}
$$

We therefore obtain that

$$
\begin{aligned}
(1- & \left.\alpha_{n}\right)\left(1-\beta_{n}\right)\left\|x_{n}-\rho_{n}\right\|^{2} \\
\leq & \left\|x_{n}-p\right\|^{2}+\alpha_{n}\|u-p\|^{2}-\left\|x_{n+1}-p\right\|^{2} \\
& +2 r_{n}\left(1-\beta_{n}\right)\left\|x_{n}-\rho_{n}\right\|\left\|A x_{n}-A p\right\| \\
\leq & \left(\left\|x_{n}-p\right\|+\left\|x_{n+1}-p\right\|\right)\left\|x_{n}-x_{n+1}\right\|+\alpha_{n}\|u-p\|^{2} \\
& +2 r_{n}\left(1-\beta_{n}\right)\left\|x_{n}-\rho_{n}\right\|\left\|A x_{n}-A p\right\| .
\end{aligned}
$$

In view of Restrictions (a), (b) and (c), we find from (2.10) that

$$
\lim _{n \rightarrow \infty}\left\|x_{n}-\rho_{n}\right\|=0
$$

Next, we show that $\limsup _{n \rightarrow \infty}\left\langle u-\bar{x}, \rho_{n}-\bar{x}\right\rangle \leq 0$, where $\bar{x}=P_{F(T) \cap(A+B)^{-1}(0)} u$. To show it, we can choose a subsequence $\left\{\rho_{n_{i}}\right\}$ of $\left\{\rho_{n}\right\}$ such that

$$
\limsup _{n \rightarrow \infty}\left\langle u-\bar{x}, \rho_{n}-\bar{x}\right\rangle=\lim _{i \rightarrow \infty}\left\langle u-\bar{x}, \rho_{n_{i}}-\bar{x}\right\rangle .
$$

Since $\rho_{n_{i}}$ is bounded, we can choose a subsequence $\left\{\rho_{n_{i j}}\right\}$ of $\left\{\rho_{n_{i}}\right\}$ which converges weakly to some point $x$. We may assume, without loss of generality, that $\rho_{n_{i}}$ converges weakly to $x$. Since $\rho_{n}=J_{r_{n}}\left(x_{n}-r_{n} A x_{n}\right)$, we find that $\frac{x_{n}-\rho_{n}}{r_{n}}-A x_{n} \in B \rho_{n}$. Since $B$ is monotone, we get, for any $(\mu, v) \in B$, that $\left\langle\rho_{n}-\mu, \frac{x_{n}-\rho_{n}}{r_{n}}-A x_{n}-v\right\rangle \geq 0$. Replacing $n$ by $n_{i}$ and letting $i \rightarrow \infty$, we obtain from (2.12) that $\langle x-\mu,-A x-v\rangle \geq 0$. This means $-A x \in B x$, that is, $0 \in(A+B)(x)$. Hence we get $x \in(A+B)^{-1}(0)$. Next, we show that $x \in F(T)$. Notice that

$$
\begin{aligned}
\left\|z_{n}-x_{n}\right\| & \leq\left\|\operatorname{Proj}_{C}\left(\alpha_{n} u+\left(1-\alpha_{n}\right) J_{r_{n}}\left(x_{n}-r_{n} A x_{n}\right)\right)-x_{n}\right\| \\
& \leq \alpha_{n}\left\|u-x_{n}\right\|+\left(1-\alpha_{n}\right)\left\|J_{r_{n}}\left(x_{n}-r_{n} A x_{n}\right)-x_{n}\right\| .
\end{aligned}
$$

In view of Restriction (a), we find from (2.12) that $\lim _{n \rightarrow \infty}\left\|z_{n}-x_{n}\right\|=0$. Note that

$$
\begin{aligned}
& \left\|\left(\gamma_{n} x_{n}+\left(1-\gamma_{n}\right) T^{n} x_{n}\right)-x_{n}\right\| \\
& \leq\left\|\left(\gamma_{n} x_{n}+\left(1-\gamma_{n}\right) T^{n} x_{n}\right)-\left(\gamma_{n} z_{n}+\left(1-\gamma_{n}\right) T^{n} z_{n}\right)\right\| \\
& \quad+\left\|\left(\gamma_{n} z_{n}+\left(1-\gamma_{n}\right) T^{n} z_{n}\right)-x_{n}\right\| \\
& \quad \leq \gamma_{n}\left\|x_{n}-z_{n}\right\|+\left(1-\gamma_{n}\right)\left\|T^{n} x_{n}-T^{n} z_{n}\right\|+\left\|\left(\gamma_{n} z_{n}+\left(1-\gamma_{n}\right) T^{n} z_{n}\right)-x_{n}\right\| \\
& \leq L\left\|x_{n}-z_{n}\right\|+\left\|y_{n}-x_{n}\right\| .
\end{aligned}
$$


It follows from $(2.8)$ that

$$
\lim _{n \rightarrow \infty}\left\|\left(\gamma_{n} x_{n}+\left(1-\gamma_{n}\right) T^{n} x_{n}\right)-x_{n}\right\|=0
$$

Note that

$$
\begin{aligned}
\left\|T^{n} x_{n}-x_{n}\right\| \leq & \left\|T^{n} x_{n}-\left(\gamma_{n} x_{n}+\left(1-\gamma_{n}\right) T^{n} x_{n}\right)\right\| \\
& +\left\|\left(\gamma_{n} x_{n}+\left(1-\gamma_{n}\right) T^{n} x_{n}\right)-x_{n}\right\| \\
\leq & \gamma_{n}\left\|T^{n} x_{n}-x_{n}\right\|+\left\|\left(\gamma_{n} x_{n}+\left(1-\gamma_{n}\right) T^{n} x_{n}\right)-x_{n}\right\| .
\end{aligned}
$$

It follows that $\left(1-\gamma_{n}\right)\left\|T^{n} x_{n}-x_{n}\right\| \leq\left\|\left(\gamma_{n} x_{n}+\left(1-\gamma_{n}\right) T^{n} x_{n}\right)-x_{n}\right\|$. This implies from Restriction (d) and (2.13) that $\lim _{n \rightarrow \infty}\left\|T^{n} x_{n}-x_{n}\right\|=0$. Since $T$ is uniformly $L$-Lipschitz continuous, we can obtain that $\lim _{n \rightarrow \infty}\left\|T x_{n}-x_{n}\right\|=0$. In view of Lemma 1.4, we find that $x \in F(T)$. This implies that

$$
\limsup _{n \rightarrow \infty}\left\langle u-\bar{x}, \rho_{n}-\bar{x}\right\rangle=\langle u-\bar{x}, x-\bar{x}\rangle \leq 0 .
$$

On the other hand, we have

$$
\begin{aligned}
\left\|x_{n+1}-\bar{x}\right\|^{2} \leq & \beta_{n}\left\|x_{n}-\bar{x}\right\|^{2}+\left(1-\beta_{n}\right)\left\|y_{n}-\bar{x}\right\|^{2} \\
\leq & \beta_{n}\left\|x_{n}-x\right\|^{2}+\left(1-\beta_{n}\right)\left\|z_{n}-\bar{x}\right\|^{2} \\
\leq & \beta_{n}\left\|x_{n}-x\right\|^{2}+\left(1-\beta_{n}\right)\left\|\alpha_{n}\left(u_{n}-\bar{x}\right)+\left(1-\alpha_{n}\right)\left(\rho_{n}-\bar{x}\right)\right\|^{2} \\
\leq & \beta_{n}\left\|x_{n}-x\right\|^{2}+\left(1-\alpha_{n}\right)^{2}\left(1-\beta_{n}\right)\left\|\rho_{n}-x\right\|^{2} \\
& +2 \alpha_{n}\left(1-\beta_{n}\right)\left\langle u-\bar{x}, \rho_{n}-x\right\rangle \\
\leq & \left(1-\alpha_{n}\left(1-\beta_{n}\right)\right)\left\|x_{n}-\bar{x}\right\|^{2}+2 \alpha_{n}\left(1-\beta_{n}\right)\left\langle u-\bar{x}, \rho_{n}-\bar{x}\right\rangle .
\end{aligned}
$$

From Lemma 1.3, we find that $\lim _{n \rightarrow \infty}\left\|x_{n}-\bar{x}\right\|=0$. This completes the proof.

If $T$ is asymptotically nonexpansive, then we find the following result.

Corollary 2.2 Let $C$ be a nonempty closed convex subset of $H$. Let $T: C \rightarrow C$ be an asymptotically nonexpansive mapping. Let $A: C \rightarrow H$ be an $\alpha$-inverse-strongly monotone mapping, and let $B$ be a maximal monotone operator on $H$. Assume that $F(T) \cap(A+B)^{-1}(0) \neq \emptyset$. Let $\left\{\alpha_{n}\right\}$ and $\left\{\beta_{n}\right\}$ be real number sequences in $(0,1)$. Let $J_{r_{n}}=\left(I+r_{n} B\right)^{-1}$, where $\left\{r_{n}\right\}$ is a positive real number sequence. Let $\left\{x_{n}\right\}$ be a sequence in $C$ generated by: $x_{1} \in C$ is chosen arbitrarily and

$$
\left\{\begin{array}{l}
z_{n}=P_{C}\left(\alpha_{n} u+\left(1-\alpha_{n}\right) J_{r_{n}}\left(x_{n}-r_{n} A x_{n}\right)\right), \\
x_{n+1}=\beta_{n} x_{n}+\left(1-\beta_{n}\right) T^{n} z_{n}, \quad \forall n \geq 1 .
\end{array}\right.
$$

Assume that the sequences $\left\{\alpha_{n}\right\},\left\{\gamma_{n}\right\}$ and $\left\{r_{n}\right\}$ satisfy the following restrictions:

(a) $0<a \leq r_{n} \leq b<2 \alpha, \lim _{n \rightarrow \infty}\left|r_{n+1}-r_{n}\right|=0$;

(b) $\lim _{n \rightarrow \infty} \alpha_{n}=0, \sum_{n=1}^{\infty} \alpha_{n}=\infty$;

(c) $0<c \leq \beta_{n} \leq d<1$, 
where $a, b, c$ and $d$ are some real numbers. If $T$ is asymptotically regular, then the sequence $\left\{x_{n}\right\}$ converges strongly to some point $\bar{x}$, where $\bar{x}=P_{F(T) \cap(A+B)^{-1}(0)} u$.

\section{Applications}

In this section, we shall consider equilibrium problems and variational inequalities.

Let $F$ be a bifunction of $C \times C$ into $\mathbb{R}$, where $\mathbb{R}$ denotes the set of real numbers. Recall the following equilibrium problem:

Find $x \in C$ such that $F(x, y) \geq 0, \quad \forall y \in C$

In this work, we use $E P(F)$ to denote the solution set of the equilibrium problem.

To study the equilibrium problems, we may assume that $F$ satisfies the following conditions:

(A1) $F(x, x)=0$ for all $x \in C$;

(A2) $F$ is monotone, i.e., $F(x, y)+F(y, x) \leq 0$ for all $x, y \in C$;

(A3) for each $x, y, z \in C$,

$$
\limsup _{t \downarrow 0} F(t z+(1-t) x, y) \leq F(x, y)
$$

(A4) for each $x \in C, y \mapsto F(x, y)$ is convex and weakly lower semi-continuous.

Putting $F(x, y)=\langle A x, y-x\rangle$ for every $x, y \in C$, we see that the equilibrium problem is reduced to the variational inequality (1.1).

The following lemma can be found in [40].

Lemma 3.1 Let $C$ be a nonempty closed convex subset of $H$, and let $F: C \times C \rightarrow \mathbb{R}$ be a bifunction satisfying (A1)-(A4). Then, for any $r>0$ and $x \in H$, there exists $z \in C$ such that

$$
F(z, y)+\frac{1}{r}\langle y-z, z-x\rangle \geq 0, \quad \forall y \in C .
$$

Further, define

$$
T_{r} x=\left\{z \in C: F(z, y)+\frac{1}{r}\langle y-z, z-x\rangle \geq 0, \forall y \in C\right\}
$$

for all $r>0$ and $x \in H$. Then the following hold:

(a) $T_{r}$ is single-valued;

(b) $T_{r}$ is firmly nonexpansive, i.e., for any $x, y \in H$,

$$
\left\|T_{r} x-T_{r} y\right\|^{2} \leq\left\langle T_{r} x-T_{r} y, x-y\right\rangle
$$

(c) $F\left(T_{r}\right)=E P(F)$;

(d) $E P(F)$ is closed and convex.

Lemma 3.2 [2] Let $C$ be a nonempty closed convex subset of a real Hilbert space $H$, let $F$ be a bifunction from $C \times C$ to $\mathbb{R}$ which satisfies (A1)-(A4), and let $A_{F}$ be a multivalued 
mapping of $H$ into itself defined by

$$
A_{F} \mathcal{X}= \begin{cases}\{z \in H: F(x, y) \geq\langle y-x, z\rangle, \forall y \in C\}, & x \in C, \\ \emptyset, & x \notin C .\end{cases}
$$

Then $A_{F}$ is a maximal monotone operator with the domain $D\left(A_{F}\right) \subset C, E P(F)=A_{F}^{-1}(0)$ and

$$
T_{r} x=\left(I+r A_{F}\right)^{-1} x, \quad \forall x \in H, r>0,
$$

where $T_{r}$ is defined as in (3.1).

The following result is not derived based on Theorem 2.1 and Lemma 3.2.

Theorem 3.3 Let $C$ be a nonempty closed convex subset of $H$. Let $T: C \rightarrow C$ be an asymptotically $\kappa$-strict pseudocontraction. Let $F$ be a bifunction from $C \times C$ to $\mathbb{R}$ which satisfies (A1)-(A4). Assume that $F(T) \cap E P(F) \neq \emptyset$. Let $\left\{\alpha_{n}\right\},\left\{\beta_{n}\right\}$ and $\left\{\gamma_{n}\right\}$ be real number sequences in $(0,1)$. Let $\left\{x_{n}\right\}$ be a sequence in $C$ generated by: $x_{1} \in C$ is chosen arbitrarily and

$$
\left\{\begin{array}{l}
w_{n} \in C \text { such that } F_{B}\left(w_{n}, u\right)+\frac{1}{r_{n}}\left\langle u-w_{n}, w_{n}-x_{n}\right\rangle \geq 0, \quad \forall u \in C, \\
z_{n}=P_{C}\left(\alpha_{n} u+\left(1-\alpha_{n}\right) w_{n}\right), \\
y_{n}=\gamma_{n} z_{n}+\left(1-\gamma_{n}\right) T^{n} z_{n}, \\
x_{n+1}=\beta_{n} x_{n}+\left(1-\beta_{n}\right) y_{n}, \quad \forall n \geq 1 .
\end{array}\right.
$$

Assume that the sequences $\left\{\alpha_{n}\right\},\left\{\beta_{n}\right\},\left\{\gamma_{n}\right\}$ and $\left\{r_{n}\right\}$ satisfy the following restrictions:

(a) $0<a \leq r_{n} \leq b<2 \alpha, \lim _{n \rightarrow \infty}\left|r_{n+1}-r_{n}\right|=0$;

(b) $\lim _{n \rightarrow \infty} \alpha_{n}=0, \sum_{n=1}^{\infty} \alpha_{n}=\infty$;

(c) $0<c \leq \beta_{n} \leq d<1$;

(d) $\kappa \leq \gamma_{n} \leq e<1, \lim _{n \rightarrow \infty}\left|\gamma_{n+1}-\gamma_{n}\right|=0$,

where $a, b, c, d$ and e are some real numbers. If $T$ is asymptotically regular, then the sequence $\left\{x_{n}\right\}$ converges strongly to some point $\bar{x}$, where $\bar{x}=P_{F(T) \cap E P(F)} u$.

If $T$ is asymptotically nonexpansive, then Theorem 3.3 is reduced to the following.

Corollary 3.4 Let $C$ be a nonempty closed convex subset of $H$. Let $T: C \rightarrow C$ be an asymptotically nonexpansive mapping. Let $F$ be a bifunction from $C \times C$ to $\mathbb{R}$ which satisfies (A1)(A4). Assume that $F(T) \cap E P(F) \neq \emptyset$. Let $\left\{\alpha_{n}\right\}$ and $\left\{\beta_{n}\right\}$ be real number sequences in $(0,1)$. Let $\left\{x_{n}\right\}$ be a sequence in $C$ generated by: $x_{1} \in C$ is chosen arbitrarily and

$$
\left\{\begin{array}{l}
w_{n} \in C \text { such that } F_{B}\left(w_{n}, u\right)+\frac{1}{r_{n}}\left\langle u-w_{n}, w_{n}-x_{n}\right\rangle \geq 0, \quad \forall u \in C, \\
z_{n}=P_{C}\left(\alpha_{n} u+\left(1-\alpha_{n}\right) w_{n}\right), \\
x_{n+1}=\beta_{n} x_{n}+\left(1-\beta_{n}\right) T^{n} z_{n}, \quad \forall n \geq 1 .
\end{array}\right.
$$

Assume that the sequences $\left\{\alpha_{n}\right\},\left\{\beta_{n}\right\},\left\{\gamma_{n}\right\}$ and $\left\{r_{n}\right\}$ satisfy the following restrictions:

(a) $0<a \leq r_{n} \leq b<2 \alpha, \lim _{n \rightarrow \infty}\left|r_{n+1}-r_{n}\right|=0$;

(b) $\lim _{n \rightarrow \infty} \alpha_{n}=0, \sum_{n=1}^{\infty} \alpha_{n}=\infty$;

(c) $0<c \leq \beta_{n} \leq d<1$, 
where $a, b, c$ and $d$ are some real numbers. If $T$ is asymptotically regular, then the sequence $\left\{x_{n}\right\}$ converges strongly to some point $\bar{x}$, where $\bar{x}=P_{F(T) \cap E P(F)} u$.

Let $H$ be a Hilbert space and $f: H \rightarrow(-\infty,+\infty]$ be a proper convex lower semicontinuous function. Then the subdifferential $\partial f$ of $f$ is defined as follows:

$$
\partial f(x)=\{y \in H: f(z) \geq f(x)+\langle z-x, y\rangle, z \in H\}, \quad \forall x \in H .
$$

From Rockafellar [41], we find that $\partial f$ is maximal monotone. It is easy to verify that $0 \in$ $\partial f(x)$ if and only if $f(x)=\min _{y \in H} f(y)$. Let $I_{C}$ be the indicator function of $C$, i.e.,

$$
I_{C}(x)= \begin{cases}0, & x \in C, \\ +\infty, & x \notin C .\end{cases}
$$

Since $I_{C}$ is a proper lower semicontinuous convex function on $H$, we see that the subdifferential $\partial I_{C}$ of $I_{C}$ is a maximal monotone operator.

Lemma 3.5 [2] Let $C$ be a nonempty closed convex subset of a real Hilbert space $H$, Proj ${ }_{C}$ the metric projection from $H$ onto $C, \partial I_{C}$ the subdifferential of $I_{C}$, where $I_{C}$ is defined above and $J_{\lambda}=\left(I+\lambda \partial I_{C}\right)^{-1}$. Then

$$
y=J_{\lambda} x \quad \Longleftrightarrow \quad y=\operatorname{Proj}_{C} x, \quad x \in H, y \in C .
$$

Now, we consider a variation inequality problem.

Theorem 3.6 Let $C$ be a nonempty closed convex subset of H. Let $T: C \rightarrow C$ be an asymptotically $\kappa$-strict pseudocontraction. Let $A: C \rightarrow H$ be an $\alpha$-inverse-strongly monotone mapping. Assume that $F(T) \cap V I(C, A) \neq \emptyset$. Let $\left\{\alpha_{n}\right\},\left\{\beta_{n}\right\}$ and $\left\{\gamma_{n}\right\}$ be real number sequences in $(0,1)$. Let $\left\{x_{n}\right\}$ be a sequence in $C$ generated by: $x_{1} \in C$ is chosen arbitrarily and

$$
\left\{\begin{array}{l}
z_{n}=P_{C}\left(\alpha_{n} u+\left(1-\alpha_{n}\right) P_{C}\left(x_{n}-r_{n} A x_{n}\right)\right), \\
y_{n}=\gamma_{n} z_{n}+\left(1-\gamma_{n}\right) T^{n} z_{n}, \\
x_{n+1}=\beta_{n} x_{n}+\left(1-\beta_{n}\right) y_{n}, \quad \forall n \geq 1 .
\end{array}\right.
$$

Assume that the sequences $\left\{\alpha_{n}\right\},\left\{\beta_{n}\right\},\left\{\gamma_{n}\right\}$ and $\left\{r_{n}\right\}$ satisfy the following restrictions:

(a) $0<a \leq r_{n} \leq b<2 \alpha, \lim _{n \rightarrow \infty}\left|r_{n+1}-r_{n}\right|=0$;

(b) $\lim _{n \rightarrow \infty} \alpha_{n}=0, \sum_{n=1}^{\infty} \alpha_{n}=\infty$;

(c) $0<c \leq \beta_{n} \leq d<1$;

(d) $\kappa \leq \gamma_{n} \leq e<1, \lim _{n \rightarrow \infty}\left|\gamma_{n+1}-\gamma_{n}\right|=0$,

where $a, b, c, d$ and e are some real numbers. If $T$ is asymptotically regular, then the sequence $\left\{x_{n}\right\}$ converges strongly to some point $\bar{x}$, where $\bar{x}=P_{F(T) \cap V I(C, A)} u$.

Proof Put $B x=\partial I_{C}$. Next, we show that $V I(C, A)=\left(A+\partial I_{C}\right)^{-1}(0)$. Notice that

$$
\begin{aligned}
x \in\left(A+\partial I_{C}\right)^{-1}(0) & \Longleftrightarrow 0 \in A x+\partial I_{C} x \\
& \Longleftrightarrow-A x \in \partial I_{C} x
\end{aligned}
$$




$$
\begin{aligned}
& \Longleftrightarrow \quad\langle A x, y-x\rangle \geq 0 \\
& \Longleftrightarrow \quad x \in V I(C, A) .
\end{aligned}
$$

\section{From Lemma 3.5, we can conclude the desired conclusion immediately.}

\section{Competing interests}

The author declares that they have no competing interests.

\section{Acknowledgements}

The author is grateful to the referees for useful suggestions which improved the contents of the article.

\section{Received: 16 December 2013 Accepted: 13 February 2014 Published: 27 Feb 2014}

\section{References}

1. Cho, SY, Qin, X, Kang, SM: Iterative processes for common fixed points of two different families of mappings with applications. J. Glob. Optim. 57, 1429-1446 (2013)

2. Takahashi, S, Takahashi, W, Toyoda, M: Strong convergence theorems for maximal monotone operators with nonlinear mappings in Hilbert spaces. J. Optim. Theory Appl. 147, 27-41 (2010)

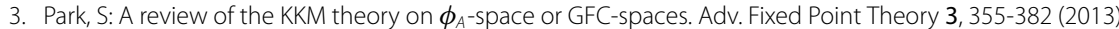

4. Zegeye, $\mathrm{H}$, Shahzad, N: Strong convergence theorem for a common point of solution of variational inequality and fixed point problem. Adv. Fixed Point Theory 2, 374-397 (2012)

5. Kim, JK: Strong convergence theorems by hybrid projection methods for equilibrium problems and fixed point problems of the asymptotically quasi- $\phi$-nonexpansive mappings. Fixed Point Theory Appl. 2011, Article ID 10 (2011)

6. Zhang, M: Iterative algorithms for common elements in fixed point sets and zero point sets with applications. Fixed Point Theory Appl. 2012, Article ID 21 (2012)

7. Zhang, M: Strong convergence of a viscosity iterative algorithm in Hilbert spaces. J. Nonlinear Funct. Anal. 2014 Article ID 1 (2014)

8. Qin, X, Cho, YJ, Kang, SM: Convergence theorems of common elements for equilibrium problems and fixed point problems in Banach spaces. J. Comput. Appl. Math. 225, 20-30 (2009)

9. Wang, Z, Lou, W: A new iterative algorithm of common solutions to quasi-variational inclusion and fixed point problems. J. Math. Comput. Sci. 3, 57-72 (2013)

10. Cho, SY, Li, W, Kang, SM: Convergence analysis of an iterative algorithm for monotone operators. J. Inequal. Appl. 2013, Article ID 199 (2013)

11. Wu, C, Liu, A: Strong convergence of a hybrid projection iterative algorithm for common solutions of operator equations and of inclusion problems. Fixed Point Theory Appl. 2012, Article ID 90 (2012)

12. Noor, MA, Noor, KI, Waseem, M: Decomposition method for solving system of linear equations. Eng. Math. Lett. 2, 34-41 (2012)

13. Cho, SY, Kang, SM: Approximation of common solutions of variational inequalities via strict pseudocontractions. Acta Math. Sci. 32, 1607-1618 (2012)

14. He, RH: Coincidence theorem and existence theorems of solutions for a system of Ky Fan type minimax inequalities in FC-spaces. Adv. Fixed Point Theory 2, 47-57 (2012)

15. Qin, X, Su, Y: Strong convergence theorems for relatively nonexpansive mappings in a Banach space. Nonlinear Anal. 67, 1958-1965 (2007)

16. Wu, C: Mann iteration for zero theorems of accretive operators. J. Fixed Point Theory 2013, Article ID 3 (2013)

17. Hecai, Y: On weak convergence of an iterative algorithm for common solutions of inclusion problems and fixed point problems in Hilbert spaces. Fixed Point Theory Appl. 2013, Article ID 155 (2013)

18. Cho, SY, Kang, SM: Approximation of fixed points of pseudocontraction semigroups based on a viscosity iterative process. Appl. Math. Lett. 24, 224-228 (2011)

19. LV, S: Generalized systems of variational inclusions involving $(A, \eta)$-monotone mappings. Adv. Fixed Point Theory 1 , 1-14 (2011)

20. Qin, X, Cho, SY, Kang, SM: Iterative algorithms for variational inequality and equilibrium problems with applications J. Glob. Optim. 48, 423-445 (2010)

21. Hecai, Y: On solutions of inclusion problems and fixed point problems. Fixed Point Theory Appl. 2013, Article ID 11 (2013)

22. Cheng, $\mathrm{P}, \mathrm{Wu}, \mathrm{H}$ : Strong convergence theorems for common solutions of a family of nonexpansive mappings and an accretive operator. Fixed Point Theory Appl. 2013, Article ID 172 (2013)

23. Qin, X, Cho, SY, Kang, SM: Convergence of an iterative algorithm for systems of variational inequalities and nonexpansive mappings with applications. J. Comput. Appl. Math. 233, 231-240 (2009)

24. Kamimura, S, Takahashi, W: Approximating solutions of maximal monotone operators in Hilbert spaces. J. Approx. Theory 106, 226-240 (2000)

25. Yang, S: Zero theorems of accretive operators in reflexive Banach spaces. J. Nonlinear Funct. Anal. 2013, Article ID 2 (2013)

26. Cho, SY, Qin, X, Kang, SM: Hybrid projection algorithms for treating common fixed points of a family of demicontinuous pseudocontractions. Appl. Math. Lett. 25, 854-857 (2012)

27. Yuan, Q: Some results on asymptotically quasi- $\phi$-nonexpansive mappings in the intermediate sense. J. Fixed Point Theory 2012, Article ID 1 (2012) 
28. Cho, SY, Kang, SM: Zero point theorems for $m$-accretive operators in a Banach space. Fixed Point Theory 13, 49-58 (2012)

29. Wu, C: Convergence of algorithms for an infinite family nonexpansive mappings and relaxed cocoercive mappings in Hilbert spaces. Adv. Fixed Point Theory 4, 125-139 (2014)

30. Kangtunyakarn, A: Iterative algorithms for finding a common solution of system of the set of variational inclusion problems and the set of fixed point problems. Fixed Point Theory Appl. 2011, Article ID 38 (2011)

31. Yang, S: Zero theorems of accretive operators in reflexive Banach spaces. J. Nonlinear Funct. Anal. 2013, Article ID 2 (2013)

32. Kim, JK, Chandok, S: Coupled common fixed point theorems for generalized nonlinear contraction mappings with the mixed monotone property in partially ordered metric spaces. Fixed Point Theory Appl. 2013, Article ID 307 (2013)

33. Qin, X, Agarwal, RP: Shrinking projection methods for a pair of asymptotically quasi- $\boldsymbol{\phi}$-nonexpansive mappings. Numer. Funct. Anal. Optim. 31, 1072-1089 (2010)

34. Luo, H, Wang, Y: Iterative approximation for the common solutions of a infinite variational inequality system for inverse-strongly accretive mappings. J. Math. Comput. Sci. 2, 1660-1670 (2012)

35. Cho, SY: Approximation of solutions of a generalized variational inequality problem based on iterative methods. Commun. Korean Math. Soc. 25, 207-214 (2010)

36. Opial, Z: Weak convergence of the sequence of successive approximation for nonexpansive mappings. Bull. Am. Math. Soc. 73, 591-597 (1967)

37. Cho, SY: Strong convergence of an iterative algorithm for sums of two monotone operators. J. Fixed Point Theory 2013, Article ID 6 (2013)

38. Liu, L: Ishikawa-type and Mann-type iterative processes with errors for constructing solutions of nonlinear equations involving $m$-accretive operators in Banach spaces. Nonlinear Anal. 34, 307-317 (1998)

39. Sahu, DR, Xu, HK, Yao, JC: Asymptotically strict pseudocontractive mappings in the intermediate sense. Nonlinear Anal. 70, 3502-3511 (2009)

40. Blum, E, Oettli, W: From optimization and variational inequalities to equilibrium problems. Math. Stud. 63, 123-145 (1994)

41. Rockafellar, RT: Characterization of the subdifferentials of convex functions. Pac. J. Math. 17, 497-510 (1966)

10.1186/1687-1812-2014-52

Cite this article as: Zhang: An algorithm for treating asymptotically strict pseudocontractions and monotone operators. Fixed Point Theory and Applications 2014, 2014:52

\section{Submit your manuscript to a SpringerOpen ${ }^{\ominus}$ journal and benefit from:}

- Convenient online submission

Rigorous peer review

- Immediate publication on acceptance

- Open access: articles freely available online

- High visibility within the field

- Retaining the copyright to your article 